\title{
Factores de riesgo para primer episodio de accidente cerebro vascular encefálico a diferentes niveles de altitud
}

\author{
Risk factors for first episode of accident encephal vascular brain at different altitude \\ levels
}

\author{
Jose Antonio Romero Cordova « 1,a,b, Aníbal Valentín Díaz Lazo 2a,a
}

Filiación y grado académico

Hospital Regional Docente Clínico Quirúrgico

Daniel Alcides Carrión, Huancayo, Perú.

Facultad de Medicina Humana. Universidad

Peruana Los Andes, Huancayo, Perú.

Medico neurólogo.

Medico internista.

(10. ORCID iD de Jose Romero

https://orcid.org/0000-0003-4723-8801

(10) ORCID iD de Aníbal Díaz

http://orcid.org/0000-0002-9282-9435

Contribución de los autores

JARC: Contribución significativa en la concepción, diseño, recolección de datos, revisión y aprobación de la versión final.

AVDL: Contribución significativa en el diseño análisis e interpretación de datos, redacción, revisión crítica y aprobación de la versión final del contenido.

Fuentes de financiamiento

La presente investigación no ha recibido ninguna fuente de financiamiento.

Conflictos de interés

Ninguno.

Recibido: 28-08-2020

Arbitrado por pares

Aceptado: 27-11-2020

Citar como

Romero Cordova JA, Díaz Lazo AV. Factores de riesgo para primer episodio de accidente cerebro vascular encefálico a diferentes niveles de altitud. Rev Peru Cienc Salud. 2020; 2(4): 225-32. doi: https://doi.org/10.37711/rpcs.2020.2.4.224

Correspondencia

Jose Antonio Romero Cordova

Email: jommed_5@yahoo.es

\section{RESUMEN}

Objetivo. Determinar los factores de riesgo para el primer episodio de accidente cerebrovascular (ACV) encefálico en personas que habitan a diferentes niveles de altitud. Métodos. Se realizó un estudio observacional, transversal y analítico en dos hospitales situados a 150 m s.n.m. y 3250 m s.n.m, entre el año 2012 y el 2018. La muestra fue seleccionada según criterios de inclusión y exclusión. El diagnostico de ACV fue determinado por tomografía o resonancia cerebral. Resultados. Se incluyeron 72 (100\%) pacientes, 40 (55,5\%) vivían a 150 m s.n.m. y $32(44,5 \%)$ a 3250 m s.n.m. Los promedios de edades fueron $64,3+-13,9$ y $71,6+-14,0$ años, respectivamente $(p=0,033)$.El grupo etario más afectado tenía entre 70 a 79 años y se encontraba con mayor porcentaje en altura (43,7 \% vs $27,5 \%)$. Los factores de riesgo con mayor frecuencia para ACV a nivel del mar fueron la hipertensión arterial $(95,0 \%$ vs. $62,5 \%)$, diabetes mellitus ( $47,5 \%$ vs. $3,1 \%$ ) y fibrilación auricular ( $15 \%$ vs $0 \%$ ) en comparación a la altura. El nivel socioeconómico bajo ( $81,2 \%$ vs $27,5 \%)$, el género femenino $(75,0 \%$ vs $42,5 \%)$, tener enfermedad valvular $(6,2 \%$ vs $2,5 \%$ ) y la policitemia ( $9,3 \%$ vs $0,0 \%$ ) fueron asociados con mayor frecuencia al ACV en altura. Conclusión. Se concluye que la hipertensión arterial es un factor de riesgo importante para el primer episodio de ACV encefálico, tanto a nivel del mar como en altura. La diabetes y la fibrilación auricular fueron más frecuentes a nivel del mar y en altura el nivel socioeconómico bajo, la policitemia y el padecer de enfermedad valvular.

Palabras clave: accidente cerebrovascular; hipertensión esencial; altitud (Fuente: DeCS-BIREME)

\section{ABSTRACT}

Objective. To determine the risk factors for the first episode of cerebral cerebrovascular accident (CVA) in people living at different levels of altitude. Methods. An observational, cross-sectional and analytical study was carried out in two hospitals located at 150 meters above sea level and 3250 meters above sea level, between 2012 and 2018, the sample was selected according to inclusion and exclusion criteria. The diagnosis of stroke was determined by tomography and / or brain resonance. Results. 72 (100\%) patients were included, 40 (55.5\%) lived at 150 meters above sea level and 32 (44.5\%) at 3250 meters above sea level; the averages of ages were $64.3+-13.9$ and $71.6+-14,0$ years respectively $(p=0.033)$. The most affected age group was between 70 and 79 years, the highest percentage in height ( $43.7 \%$ vs $27.5 \%$ ). The most frequent risk factors for stroke at sea level were arterial hypertension (95.0\% vs $62.5 \%$ ), diabetes mellitus (47.5\% vs $3.1 \%$ ) and atrial fibrillation ( $15.0 \%$ vs $0.0 \%)$ compared to altitude. The low socioeconomic level $(81.2 \%$ vs $27.5 \%)$, the female gender (75.0 \% vs $42.5 \%)$, having valve disease $(6.2 \%$ vs $2.5 \%$ ) and polycythemia ( $9.3 \%$ vs $0.0 \%$ ) were more frequently associated with stroke in height. Conclusions. It is concluded that arterial hypertension is an important risk factor for the first episode of brain stroke both at sea level and at altitude. Diabetes and atrial fibrillation were at sea level and in altitude, the low socioeconomic level, polycythemia, and suffering from valve disease. Keywords: stroke; essential hypertension; altitude (Source: MeSH - NLM). 


\section{INTRODUCCIÓN}

El accidente cerebrovascular (ACV) es un déficit neurológico atribuido a una lesión del sistema nervioso central por infarto cerebral, hemorragia intracerebral y hemorragia subaracnoidea ${ }^{(1)}$. Cada año, 15 millones de personas alrededor del mundo padecen de ictus, resultando en 5 millones de muertes y 5 millones de discapacidad (2). El ACV es una de las causas más importantes de mortalidad y discapacidad en el mundo ${ }^{(3)}$. Además, es la tercera causa de muerte en mujeres y la quinta en varones ${ }^{(2)}$. En los países en desarrollo de medianos y bajos ingresos económicos se ha incrementado la incidencia de $A C V^{(3,4)}$.

Por otro lado, aproximadamente 400 millones de personas viven a más de 1500 m.s.n.m. ${ }^{(5)}, 140$ millones a más de 2500 m.s.n.m., y 35 millones aproximadamente en los andes sudamericanos y la mayor densidad poblacional se encuentra por encima de los 3500 m.s.n.m. ${ }^{(6)}$. La hipoxia a grandes altitudes desafía la capacidad del ser humano para vivir y reproducirse ${ }^{(7)}$. A pesar de esta situación existen gran cantidad de personas que habitan en los andes peruanos exhibiendo adaptaciones anatómicas, circulatorias, respiratorias, hematológicas y metabólicas al frío y a los niveles bajos de oxígeno ${ }^{(5,7)}$. En algunos pacientes se desarrolla el mal de montaña crónico cuando se desadaptan a la altura; estos presentan hipertensión pulmonar, moderada a severa, con hipoxemia acentuada y policitemia exagerada ${ }^{(6)}$. Vivir a grandes alturas y estar expuesto a un grado significativo de hipoxia hipobárica se asocia con el incremento en el riesgo de presentar un $A C V$, especialmente de tipo isquémico ${ }^{(8)}$. Existen datos del incremento de la prevalencia del ACV en personas que habitan a grandes alturas debido a la presencia de policitemia ${ }^{(8)}$.

También se reporta que la hipertensión arterial, diabetes mellitus, el tabaquismo, los factores psicosociales, el consumo de alcohol están relacionados con la frecuencia de accidentes cerebrovasculares ${ }^{\left({ }^{9}\right)}$. En este contexto, se diseñó un estudio para determinar los factores de riesgo asociados al primer episodio de accidente cerebrovascular encefálico en personas que habitan a diferentes niveles de altitud, en la ciudad de Lima y Huancayo.

\section{METODOS}

\section{Diseño y población}

El diseño del estudio fue observacional, transversal, prospectivo y analítico. El periodo de estudio comprendió de enero del 2012 a marzo del 2018. La población estuvo conformada por todos los pacientes hospitalizados en el
Servicio de Emergencia del Hospital Regional Docente Clínico Quirúrgico (HRDCQ) Daniel Alcides Carrión de Huancayo, ubicado a 3,250 m s.n.m. y en el Servicio de Neurología del Hospital Nacional 2 de mayo de Lima, situado a $150 \mathrm{~m}$ s.n.m. La muestra se determinó mediante los criterios de inclusión y exclusión. Los criterios de inclusión fueron: tener diagnóstico de ACV encefálico confirmado por tomografía o resonancia cerebral, poseer consentimiento informado para participar en el estudio, tener un primer episodio del ACV, ser varón o mujer, pacientes con localización de ACV encefálica, tener 20 a más años de edad. Los criterios de exclusión fueron: no aceptar participar en el estudio, tener dos o más episodios de ACV, tener diagnóstico de traumatismo encéfalo craneano.

\section{Variables de estudio}

Factor de riesgo: es la característica biológica o habito que permite identificar a un grupo de personas con mayor probabilidad que el resto de la población general para presentar una determinada enfermedad a lo largo de su vida ${ }^{(10)}$.

Accidente cerebrovascular encefálico (ACV): es una disfunción cerebral focal de comienzo agudo y una duración superior a 24 horas como resultado del deterioro de la circulación sanguínea ${ }^{(1)}$.

Accidente cerebovascular isquémico: interrupción de la circulación cerebral por obstrucción de la arteria (1). Accidente cerebrovascular hemorrágico: colapso de la circulación cerebral por extravasación de sangre de la arterial ${ }^{\left({ }^{10)}\right.}$. Nivel socioeconómico: es la jerarquía que tiene una persona y fue medido según el nivel de ingreso, educación y ocupación ${ }^{(11)}$.

\section{Factores de riesgo cardiovascular:}

Hipertensión arterial (HTA): Se consideró hipertensión arterial cuando las cifras de presión arterial (PA) reportaron $>140 / 90 \mathrm{mmHg}$ o tuvieron diagnóstico previo de hipertensión arterial y recibían tratamiento farmacológico antihipertensivo ${ }^{(12)}$. Edad: varones $>$ a 55 años y mujeres $>65$ años de edad ${ }^{(12)}$. Dislipidemia: cuando los valores de colesterol total eran > a $190 \mathrm{mg} / \mathrm{dl}$, colesterol LDL $>115 \mathrm{mg} / \mathrm{dl}$, Colesterol HDL $<40 \mathrm{mg} / \mathrm{ml}$ en varones y < a $46 \mathrm{mg} / \mathrm{dl}$ en mujeres, o triglicéridos $>150 \mathrm{mg} / \mathrm{dl}^{(12)}$. Obesidad. fueron considerados con índice de masa corporal $>30 \mathrm{~kg} / \mathrm{m} 2{ }^{(12)}$. Diabetes mellitus: glicemia en ayunas $>126 \mathrm{mg} / \mathrm{dl}, \mathrm{HbA} 1 \mathrm{c}>7 \%$

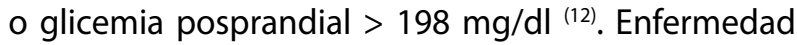
valvular: cuando presenta estenosis o insuficiencia mitral o aórtica mediante estudio ecocardiográfico ${ }^{(13)}$. Tabaquismo: fumar 1 cigarrillo o más por día durante el 
último año ${ }^{(14)}$. Ingesta de alcohol: consumir $50 \mathrm{ml}$ o más por un año ${ }^{(14)}$. Eritrocitosis excesiva (policitemia): nivel de hemoglobina > a 18g/dl en mujeres y > a $19 \mathrm{~g} / \mathrm{dl}$ en varones ${ }^{(15)}$.

Procedimiento: Previa firma del consentimiento informado se procedió a la recolección de datos a través de una entrevista al paciente $o$ a sus familiares; además se obtuvieron datos de historia clínica, los cuales fueron consignados en un cuestionario. Así mismo, el diagnóstico por imágenes se realizó a través de una tomografía cerebral sin contraste o resonancia cerebral, la cual se solicitó al paciente al ser admitido en el hospital.

Análisis estadístico: Se realizó el análisis estadístico descriptivo con frecuencias y medias, el análisis inferencial con $X^{2}$ para variables cualitativas, $t$ student para variables cuantitativas, y la asociación del factor de riesgo con odds ratio (OR) e intervalo de confianza al $95 \%$. Se consideró significativo un $\mathrm{p}<0,05$.

Aspectos éticos: Se obtuvo el consentimiento informado de cada participante. Los datos recolectados durante el estudio se utilizaron solo para fines de investigación y fueron en todo momento manejados como confidenciales.

\section{RESULTADOS}

Se incluyeron 72 (100\%) pacientes, 31(43,1 \%) varones y $41(46,9 \%)$ mujeres con un promedio de edad de $65.59+-$ 14.4 años; 40 (55,5\%) vivían a nivel del mar, 150 m.s.n.m.,

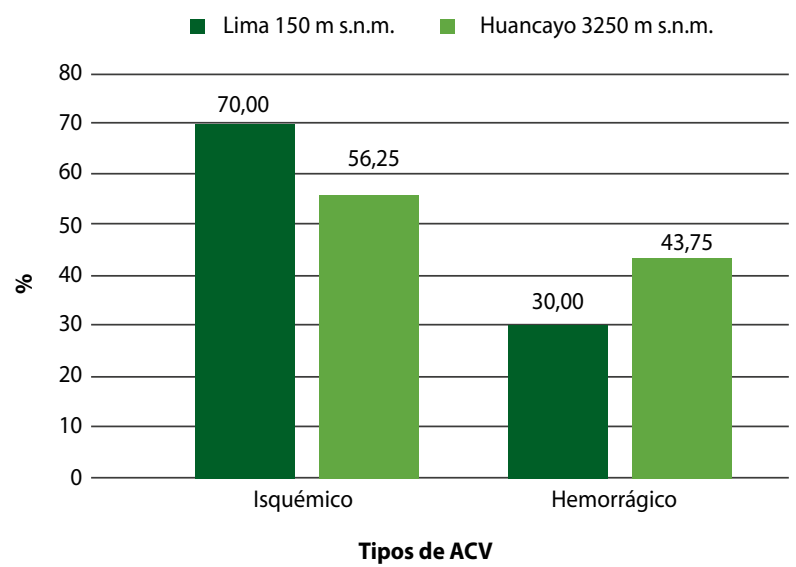

Figura 1. Tipos de $A C V$ a diferentes niveles de altitud

y $32(44,5 \%)$ a 3250 msnm, cuyos promedios de edades fueron $64,3+-13,9$ y $71,6+-14.0$ años respectivamente $(p=0,033)$. En la tabla 1 , se muestra que la frecuencia de ACV es menor por debajo de los 60 años (25\%), incrementándose a partir de la sexta década de la vida (75\%), tanto en los que viven a nivel de mar $(67,5 \%)$ así como también en quienes residen a 3250 m.s.n.m. (84,37\%), con picos altos entre los 70 a 79 años de edad en ambas ciudades con mayor porcentaje en la altura $(43,7 \%$ vs. $27,5 \% ; p>0,05)$.

En la figura 1, se muestra que el $A C V$ isquémico fue más frecuente a nivel del mar (70\%) en comparación a una ciudad de altura $(56,25 \%)$, mientras que en el caso del ACV hemorrágico la frecuencia de casos fue mayor en altura $(43,75 \%)$ que a nivel de mar (30\%).

Tabla 1. ACV y grupo etario en pacientes que habitan a diferentes niveles de altitud 2012-2018

\begin{tabular}{|c|c|c|c|c|c|c|}
\hline \multirow{3}{*}{$\begin{array}{l}\text { Grupo etario } \\
\text { (años) }\end{array}$} & \multicolumn{4}{|c|}{ Nivel de altura sobre el nivel del mar (m.) } & \multirow{3}{*}{ OR, IC (95 \%) } & \multirow{3}{*}{$p$} \\
\hline & \multicolumn{2}{|c|}{$\begin{array}{l}\text { Lima } \\
150 \mathrm{~m} .\end{array}$} & \multicolumn{2}{|c|}{$\begin{array}{l}\text { Huancayo } \\
3250 \mathrm{~m} \text {. }\end{array}$} & & \\
\hline & fi & $\%$ & fi & $\%$ & & \\
\hline 20-29 & 1 & 2,5 & 0 & 0,0 & & \\
\hline 30-39 & 0 & 0,0 & 1 & 3,13 & & \\
\hline $40-49$ & 7 & 17,5 & 2 & 6,25 & $0,31(0,06-1,63)$ & $p=0,151$ \\
\hline $50-59$ & 5 & 12,5 & 2 & 6,25 & $0,46(0,08-2,58)$ & $p=0,373$ \\
\hline 60-69 & 10 & 25,0 & 4 & 12,5 & $0,42(0,12-1,42)$ & $p=0,183$ \\
\hline 70-79 & 11 & 27,5 & 14 & 43,75 & $2,05(0,76-5,48)$ & $p=0,150$ \\
\hline 80-89 & 6 & 15,0 & 7 & 21,87 & $1,58(0,47-5,30)$ & $p=0,451$ \\
\hline $90-99$ & 0 & 0,0 & 2 & 6,25 & & \\
\hline Total & 40 & 100,0 & 32 & 100,0 & & \\
\hline
\end{tabular}

Fuente: ficha de recolección de datos, 2012-2018. 
Tabla 2. Factores de riesgo para primer episodio de accidente cerebrovascular encefálico en pacientes que habitan a diferentes niveles de altitud, 2012-2018

\begin{tabular}{|c|c|c|c|}
\hline \multirow{2}{*}{ Variable } & \multicolumn{2}{|c|}{$\begin{array}{c}\text { Nivel de altura sobre el } \\
\text { nivel del mar (m) }\end{array}$} & \multirow[b]{2}{*}{$p$} \\
\hline & $\begin{array}{c}150 \mathrm{~m} \\
\mathrm{n}(\%)\end{array}$ & $\begin{array}{c}3250 \mathrm{~m} \\
\mathbf{n}(\%)\end{array}$ & \\
\hline Edad > 60 años & $27(67,5)$ & $27(84,3)$ & $p=0,100$ \\
\hline \multicolumn{4}{|l|}{ Género } \\
\hline Masculino & $23(57,5)$ & $8(25,0)$ & $p=0,005$ \\
\hline Femenino & $17(42,5)$ & $24(75,0)$ & $p=0,005$ \\
\hline \multicolumn{4}{|l|}{ Raza } \\
\hline Mestiza & $32(80,0)$ & $31(96,8)$ & $p=0,031$ \\
\hline Negra & $6(15,0)$ & $0(0,0)$ & $p=0,022$ \\
\hline Blanca & $2(5,0)$ & $1(3,2)$ & $p=0,692$ \\
\hline \multicolumn{4}{|l|}{ Nivel socioeconómico } \\
\hline Bajo & $11(27,5)$ & $26(81,2)$ & $p=0,000$ \\
\hline Medio & $29(72,5)$ & $6(28,8)$ & $p=0,000$ \\
\hline \multicolumn{4}{|l|}{ Antecedente } \\
\hline Hipertensión arterial & $38(95,0)$ & $20(62,5)$ & $p=0,000$ \\
\hline Diabetes Mellitus & $19(47,5)$ & $1(3,1)$ & $p=0,000$ \\
\hline Fibrilación auricular & $6(15,0)$ & $0(0,0)$ & $p=0,022$ \\
\hline Dislipidemia & $4(10,0)$ & $3(9,3)$ & $p=0,929$ \\
\hline Enfermedad valvular & $1(2,5)$ & $2(6,2)$ & $p=0,428$ \\
\hline Alcoholismo & $1(2,5)$ & $1(3,1)$ & $p=0,872$ \\
\hline Policitemia & $0(0,0)$ & $3(9,3)$ & $p=0,047$ \\
\hline \multicolumn{4}{|l|}{ Horario de presentación } \\
\hline 6 a.m. - 11 a.m. & $20(50,0)$ & $13(40,6)$ & $p=0,427$ \\
\hline $12 m .-5 p, m$ & $14(35,0)$ & $8(25,0)$ & $p=0,360$ \\
\hline 6 p.m. - 11 a.m. & $4(10,0)$ & $5(15,6)$ & $p=0,473$ \\
\hline 12 m. -5 a.m. & $2(5,0)$ & $6(18,8)$ & $p=0,065$ \\
\hline
\end{tabular}

Fuente: ficha de recolección de datos, 2012-2018.

En la tabla 2, se muestra que el ACV en altura es más frecuente en personas de 60 o más años de edad, en mujeres, raza mestiza, nivel socioeconómico bajo y el antecedente de enfermedad valvular, mientras que el $\mathrm{ACV}$ a nivel del mar estuvo asociado con mayor frecuencia al sexo masculino, nivel socioeconómico medio, el antecedente de hipertensión arterial, diabetes mellitus y fibrilación auricular. La hora de mayor presentación estuvo comprendido entre las 6 a.m. a 11 a.m. con 33 (45,8 \%) casos, seguido de los 22 (32,5\%) casos de 12 m. a 5 p.m. El ACV fue más común durante el día en comparación con lo que se presentó durante la noche ( $88,3 \%$ vs. $11,7 \%)$.

En la tabla 3, se muestra que existe un mayor porcentaje de ACV localizado en el hemisferio del lado derecho (en quienes habitan en la altura en comparación a
Tabla 3. Localización del accidente cerebrovascular en pacientes que habitan a diferentes niveles de altitud, 2012-2018

\begin{tabular}{lcc}
\hline Localización hemisférica & $\begin{array}{c}\text { Lima } \\
\mathbf{1 5 0} \mathbf{~} \\
\mathbf{n}(\%)\end{array}$ & $\begin{array}{c}\text { Huancayo } \\
\mathbf{3 2 5 0} \mathbf{~} \\
\mathbf{n}(\%)\end{array}$ \\
\hline $\begin{array}{l}\text { Hemisférica } \\
\text { Derecha }\end{array}$ & $24(60,0)$ & $22(68,75)$ \\
Izquierda & $16(40,0)$ & $10(31,25)$ \\
Anatómica & & \\
Ganglios basales & $6(15,0)$ & $12(37,5)$ \\
Temporoparietal & $29(72,5)$ & $11(34,37)$ \\
Temporal & $0(0,0)$ & $3(9,37)$ \\
Parietal & $3(7,5)$ & $2(6,25)$ \\
Talámico & $1(2,5)$ & $2(6,25)$ \\
Cápsula interna & $1(2,5)$ & $1(3,13)$ \\
Temporooccipital & $1(2,5)$ & $1(3,13)$ \\
\hline Fuente: ficha de recolección de datos, 2012-2018. & &
\end{tabular}

los que viven a nivel del mar (60\%), pero esta no es significativa. La lesión neurológica estuvo localizada en mayor porcentaje a nivel de la región temporo parietal con $40(55,5 \%)$ casos, siendo más frecuente a nivel del $\operatorname{mar}(72,5 \%$ vs. $34,37 \% ; p<0,05)$ en relación a la altura, siendo en esta ultima la localización más común a nivel de los ganglios basales ( $37,5 \%$ vs. $15 \% ; p<0,05)$.

\section{DISCUSIÓN}

La incidencia del ACV reportada fue de 24,6 por 100 000 personas al año, siendo 0,10 en menores de 45 años, incrementándose a 9,6 en mayores de 85 años y la mortalidad al mes fue de $40,4 \%{ }^{(16)}$. En el Perú se ha reportado una prevalencia de $6,8 \%$ en la zona urbana y $2,7 \%$ en la zona rural en mayores de 65 años ${ }^{(4)}$. Evaluando 752 pacientes con ACV, la tasa de sobrevida al año fue de $86,4 \%$, fallecieron al año $33,3 \%$, después de un ACV inicial, el $18,6 \%$ por ACV recurrente y el $16,7 \%$ debido a comorbilidades cardiovasculares, el $14,7 \%$ por condiciones no vasculares y el $16,7 \%$ por causas no determinadas ${ }^{(17)}$. En otro estudio se reporta que la enfermedad cardiovascular puede producir hasta 19,7 \% de mortalidad hospitalaria, la mayoría durante los tres primeros días ${ }^{(3,18)}$. Ahora bien es probable que la falta de conocimiento de los factores riesgo evidenciada en nuestro medio determina la atención no oportuna del paciente.

La incidencia del ACV hemorrágico fue de un 24,2\% en personas de raza blanca, un $22,9 \%$ en personas de raza negra, 19,6\% en las personas hispanas y un 51,8\% en personas asiáticas; además, la incidencia del ictus se incrementó con la edad y no existe disminución entre 1980 y $2006^{(16)}$. 
Diversos estudios reportan el promedio de edad de las personas con riesgo de padecer enfermedad cerebrovascular. En Perú, Castañeda-Guarderas et al. reportan un promedio de $64,9+-16,9$ años ${ }^{(18)}$. En Colombia, Machado -Alva et al. reportan 64,9+- 10,9 años ${ }^{(19)} y$ en Chile, Prat reporta una media de 66 años ${ }^{(20)}$. En su casuística Dhiman et al. encontraron el promedio de edad de 62 años $^{(21)}$. Castillo-Guerra et al., evaluando 98 pacientes con $\mathrm{ACV}$ isquémico 60 varones y 38 mujeres, reportan un promedio de edad de 70,5 +- 11 años ${ }^{(22)}$. El ACV isquémico se presentó, en promedio, a la edad de 66 años en los varones, mientras que en las mujeres a los 70 años ${ }^{(23)}$. En nuestra casuística, el promedio de edad fue de 65 años, valor similar a estudios previos.

La forma más frecuente de ACV que se reporta es el isquémico, con el $48,1 \%$ de los casos, seguido del hemorrágico, con el $24,9 \%$ y la causa más común de mortalidad fue la de tipo hemorrágico con el $31,8 \%$ de los casos ${ }^{(18)}$. Otro estudio evaluando 80 casos con ACV encontró una media de 64,92 +- 11,58 años; el 73,75\% presentó isquemia cerebral y el $26,25 \%$ hemorragia intracerebral ${ }^{(24)}$. Dhiman et al., en su estudio realizado a 2000 m s.n.m., en 235 (100\%) pacientes reportan 173 (74 \%) casos de ACV isquémico y 62 ( $26 \%$ ) casos de ACV hemorrágico (21). En nuestro estudio encontramos en altura ACV isquémico en $56,25 \%$ y hemorrágico en $43,75 \%$.

La edad es un factor importante de aparición del ictus; después de los 55 años, por cada década que se incrementa la vida se duplica la incidencia del ictus; así mismo, los de mayor edad tienen una mayor mortalidad ${ }^{(23)}$. En su serie, Dhiman et al. encontraron una mayor incidencia ( $28 \%$ ) entre los 61 y los 70 años de edad ${ }^{(21)}$. Según Pratt, el $93 \%$ de los infartos cerebrales nuevos se producen en mayores de 45 años ${ }^{(20)}$. Nosotros encontramos un incremento de la presentación a partir de los 60 años de edad (75\%) con elevado porcentaje entre los 70 y los 79 años, mientras que el $84,7 \%$ tuvieron una edad de 50 o más años.

Existen factores de riesgos mayores modificables para enfermedad cardiovascular como son la hipertensión arterial, diabetes mellitus, tabaquismo y dislipidemia ${ }^{(25)}$. Cantu-Brito et al., en su estudio incluyeron 1376 pacientes de 59 hospitales, entre enero del 2005 y junio del 2006, con edad promedio de 67,5 años; los principales factores de riesgo que encontraron fueron: hipertensión $64 \%$, obesidad $51 \%$ y diabetes $35 \%{ }^{(26)}$. Dhiman et al. (2018), evaluando 235 (100\%) pacientes reportaron que los factores de riesgo asociados al ACV fueron: hipertensión arterial (66\%), tabaquismo (36\%), diabetes (14\%), fibrilación auricular (11\%), enfermedad valvular reumática (5\%) ${ }^{(21)}$.
Los factores de riesgo para DCV hemorrágico fueron la edad $(\mathrm{OR}=1,97)$, ingesta de alcohol $(\mathrm{OR}=$ $3,36)$, hipertensión arterial $(O R=3,68)$, sexo masculino $(O R=3,73)$, fumador $(O R=1,31)$ y diabetes $(O R=1,3)^{(27)}$. Investigación previa reporta que los factores de riesgo para ACV isquémico fueron: HTA (61\%), tabaquismo (36\%), diabetes (16\%), fibrilación auricular (14\%) y enfermedad valvular reumática (7 \%) y para ACV hemorrágico HTA (79\%), tabaquismo $35 \%$, diabetes (10\%), fibrilación auricular (3\%) e insuficiencia cardiaca (3\%) ${ }^{(21)}$. CastilloGuerra et al., evaluando 98 pacientes con ACV isquémico reportaron que los factores de riesgo fueron: HTA $(68,3 \%)$, hipercolesterolemia $(44,8 \%)$, diabetes $(38,7 \%)$, tabaquismo $\left(24,8 \%\right.$ y fibrilación auricular $(19,3 \%)^{(22)}$. En nuestro estudio encontramos que la hipertensión arterial, diabetes mellitus y la fibrilación fueron los factores de riesgo más frecuente para el ACV.

En una serie de pacientes con ACV isquémico se reportó que la presión arterial sistólica promedio fue de $136+-20 \mathrm{mmHg}$ y la presión arterial diastólica 78,7 $+-11,8 \mathrm{mmHg}^{(22)}$. La hipertensión arterial representa el factor más importante para la aparición ACV isquémico y hemorrágico; el incremento de los niveles de presión arterial es observado en los pacientes con ACV hemorrágico intracerebral en más del $70 \%$ de casos con cifras de presión arterial sistólica mayor a $140 \mathrm{mmHg}$ y en más del $20 \%$ por encima de $180 \mathrm{mmHg}$, lo cual está relacionado con mal pronóstico ${ }^{(28,29,30)}$. Semanas previas al ACV hemorrágico existe aumento de la presión arterial en comparación a los pacientes que desarrollan ACV isquémico, donde la presión arterial antes del evento es baja en comparación a los niveles de presión arterial posterior al evento isquémico ${ }^{(29)}$. Sare et al. reportan que la presión arterial elevada está presente en el $80 \%$ de los pacientes con $\mathrm{ACV}$ isquémico ${ }^{(30)}$. La reducción intensa de la presión arterial parece estar asociada con la atenuación del crecimiento absoluto del tamaño del hematoma dentro de las 24 horas posteriores al inicio del cuadro clínico ${ }^{(31)}$. En nuestra serie encontramos elevada frecuencia de pacientes con hipertensión arterial, tanto a nivel del mar como en altura; probablemente esta entidad clínica este asociada a una mayor presentación de ACV hemorrágico en altura.

Sin embargo, varios estudios han reportado asociaciones positivas entre el índice de masa corporal (IMC) y los riesgos de $A C V$ isquémico y global ${ }^{(32)}$. El IMC elevado aumenta linealmente el riesgo de ACV global y por subtipos (isquémico y hemorrágico), así como la mortalidad por ACV en adultos chinos ${ }^{(32)}$. En otro estudio, la adiposidad se asoció fuertemente con el ACV isquémico principalmente a través de su efecto sobre a 
presión arterial ${ }^{(33)}$. En mujeres de Gran Bretaña, el mayor IMC está asociado con el incremento del ACV isquémico pero no necesariamente con el mayor riesgo de ACV hemorrágico ${ }^{(34)}$. En nuestro estudio no evaluamos esta característica pero asumimos que, por la elevada frecuencia de hipertensos y diabéticos encontrados, muchos de nuestros pacientes deben presentar sobrepeso y obesidad porque estas entidades están relacionadas entre sí.

La hiperglicemia confiere un mayor riesgo de ACV; los diabéticos son más susceptibles a enfermedades de los pequeños vasos sanguíneos, ocasionando ACV de tipo isquémico la cual cursa con pobre pronóstico ${ }^{(25)}$. La prediabetes, así como la diabetes mellitus (DM) son factores de riesgo para el ACV ${ }^{(25)}$. Además, la DM es un factor de riesgo para el ACV isquémico y hemorrágico. El riesgo atribuido al ACV isquémico es de 2,27 (1,95$2,65)$; para el hemorrágico $1,56(1,19-2,05)$ y para el ACV no clasificado es $1,84(1,59-2,13)^{(25)}$. Se estimó que para el año 2010, unos 285 millones de personas padecerían de DM y el número proyectado para el 2030 es de 439 Millones, incremento que está atribuido a la obesidad ${ }^{(25)}$. En nuestra serie, la incidencia de la DM fue alta a nivel del mar; casi 1 de cada 2 casos reportaron DM a comparación de aquellos en la altura donde solo fue presentado por 1 caso.

La fibrilación auricular es la arritmia más frecuente con prevalencia que oscila entre 1 a $3 \%$ de la población general ${ }^{(35,36)}$; se incrementa con la edad, afectando al $7 \%$ de los individuos mayores de 65 años y alcanzando entre el 15 a $20 \%$ entre los octogenarios ${ }^{(35)}$. En la aurícula izquierda se forma el trombo debido a la pérdida de la contractibilidad auricular, estasis sanguínea, activación de la coagulación y a las características anatómicas de la orejuela izquierda ${ }^{(35)}$. En aquellos pacientes que presentan fibrilación auricular no valvular, en más del $90 \%$ de los casos la localización del trombo se encuentra en la orejuela izquierda, el riesgo anual para el ACV es de $5 \%$ y la ocurrencia es 5 veces más común en comparación a la población general ${ }^{(36,37,38)}$. En nuestra casuística encontramos fibrilación auricular en el $15 \%$ de pacientes que residían a nivel del mar.

Los ACV causados por ruptura vascular son relativamente menos frecuente que los de tipo isquémico en los pobladores de altura ${ }^{(39)}$. En nuestra serie no encontramos esta relación. El ACV de tipo isquémico es de presentación más frecuente que el de tipo hemorrágico, dado que ocupa el $70 \%$ de los casos de ACV, pero el evento isquémico se incrementa entre un 10 y un $20 \%$ en las grandes alturas que a nivel del mar ${ }^{(8)}$; lo cual estaría en relación directa con el aumento de la policitemia ${ }^{(8)}$. Otro factor de riesgo para el ACV es la estasis sanguínea causada por el frío, la inmovilización prolongada especialmente en los montañistas y la deshidratación ${ }^{(8)}$. El tiempo de exposición a alturas muy elevadas desencadena la policitemia ${ }^{(5)}$. La variable policitemia tuvo pocos casos en el estudio, siendo esta una limitación para hacer inferencias y poder generalizar.

Existen estudios que han asociado el bajo nivel de ingreso económico y de educación con la elevada mortalidad por enfermedades cardiovasculares ${ }^{(5)}$. En el estudio de Withehal se reporta que los hombres con nivel socio económico bajo tienen 2,7 veces más riesgo de mortalidad a los 10 años por enfermedad cardiovascular en comparación al nivel alto; de modo que, ajustando los factores de riesgo tradicional esta disminuye a $2.1^{(40)}$.

La limitación del estudio fue el reducido número de la muestra, lo no permite realizar generalizaciones a otros contextos, por lo que consideramos necesario realizar más estudios al respecto. Además, se concluye que la hipertensión arterial es un factor de riesgo importante para el primer episodio de ACV encefálico, tanto a nivel del mar como en altura. La DM y la fibrilación son factores de riesgo predominantemente a nivel del mar y en altura, así como el nivel socioeconómico bajo, la policitemia y el padecer de enfermedad valvular. Por último, el incremento de la edad se relaciona con mayor frecuencia de ACV.

\section{REFERENCIAS}

1. Sacco RL, Kasner SE, Broderick JP, Caplan LR, Connors JJ, Culebras A, et al. An Updated definition of stroke for the 21st Century: a statement for health care professionals from the American heart Association/American stroke Association. Stroke.2013;44(7): 2064-89 doi: 10.1161/ STR.0b013e318296aeca

2. Roy-O'Reilly M, Mac Cullough L. Sex differences in stroke. The contribution of coagulations. Exp Neurol. 2014; 259: 16-27 [Consultado el 05/01/2018]. doi: 10.1016/j.expneurol.2014.02.011

3. Málaga G, De la Cruz-Saldaña T, Busta-Flores P, Carbajal A, Santiago-Mariaca K. La enfermedad cerebrovascular en el Peru: estado actual y perspectivas de investigación clínica. Acta Med Peru. 2018; 35(1): 51-4. [Consultado el 05/01/2018]. Disponible en: http://www.scielo.org.pe/ pdf/amp/v35n1/a08v35n1.pdf

4. Davalos LF, Malaga G. El accidente cerebrovascular en el Perú. Una enfermedad prevalente olvidada y desatendida. Rev peru med exp salud publica.2014;31(2):400-401 [Consultado el 05/01/2018]. Disponible en: http:// www.scielo.org.pe/scielo.php?script=sci_arttext\&pi$d=S 1726-46342014000200040 \&$ Ing=es.

5. Bhatnagar A. Environmental determinants of cardiovascular disease. Circ Res. 2017 Jul 7; 121(2): 162-180 doi: 10.1161/CIRCRESAHA.117.306458

6. Peñaloza $D$, Arias-Stella J. The heart and pulmonary circulation at high altitudes: healthy highlanders and 
chronic mountain sickness. Circulation. 2007; 115(9): 1132-46 [Consultado el 05/01/2014]. disponible en: doi: 10.1161/CIRCULATIONAHA.106.624544.

7. Bigham AW, Wilson MJ, Julian CG, Kiyamu M, Vargas E, León-Velarde, et al. Andean an Tibetan patterns of adaptation to high altitude. Am J Hum Biol. 2013;25(2):190-7 doi: 10.1002/ajhb.22358. Epub 2013 Jan 24.

8. Ortiz E, Ojeda O, Silva F. Accidente cerebro vascular en poblaciones situadas a grandes alturas: revisión y análisis de los factores de riesgo. Rev Ecuat Neurol.2008; 17(1-3):1-21 [Consultado el 05/01/2015].disponible en: http://revecuatneurol.com/magazine_issue_article/accidente-cerebrovascular-en-poblaciones-situadas-a-grandes-alturas-revision/

9. O’Donnell MJ, Siu L, Rangarajan S, Xavier D, Liu L, Zhang $\mathrm{H}$, et al. Global and Regional effects of potentially modificable risk factors associated with acute stroke in 32 countries (INTERSTROKE): a case control study. Lancet 2016;38(10046):761-75 doi: 10.1016/S01406736(16)30506-2.

10. Marcotti AC. Estudo dos efeitos da dominancia hemisférica cerebral esquerda sobre o acidente vascular encefálico a través de tres escalas de funcionalidade. Monografias do curso fisioterapia da unioeste. 2009. 1 [Consultado el 05/01/2015]. disponible en: https:// www.efdeportes.com/efd142/acidente-vascular-encefalico-tratamento-cinesioterapeutico.htm

11. Vera-Romero OE, Vera-Romero FM. Evaluación del nivel socioeconómico: presentación de una escala adaptada en una población de Lambayeque. Rev Cuerpo Med. HNAAA.2013;6(1): 41-45 [Consultado el 05/01/2016]. disponible en: https://dialnet.unirioja.es/servlet/articulo?codigo $=4262712$

12. Mancia G, Fagard R, Narkiewicz K, Redón J, Zanchetti A, Böhm M, et al. 2013 ESH/ESC Guidelines for the management of arterial hypertension. The taske force for the management of arterial hypertension of the European Society of Hypertension (ESH) and of the European Society of Cardiology. J Hypertension. 2013; 31(7 ):1281-1357 [Consultado el 05/02/2017]. disponible en: https://academic.oup.com/eurheartj/article/34/28/2159/451304

13. Nishimura RA, Otto $C$, Bonow R, Carabello B, Erwin J, et al. 2014 AHA/ACC Guideline for the management of patients with valvular Heart Disease: Executive Summary. A Report of The American College of Cardiology / American Heart Association Task Force on Practice Guidelines. Circulation 2014; 1-96 [Consultado el 05/02/2017]. doi: $10.1161 /$ CIR.0000000000000029

14. Tsai CF, Anderson N, Thomas B, Sudlow CL. Comparing Risk Factor Profiles between Intracerebral Hemorrhage and Ischemic Atroke in Chinese and White Populations: Systematic Review and Meta-Analysis. Plos One.2016;11(3):eo151743 [Consultado el 05/02/2017]. https://doi.org/10.1371/journal.pone.0151743

15. Amaru-Lucanu R, Vera-Carrasco O. Guia para el diagnóstico y tratamiento de las eritrocitosis patológicas en la altura. Rev Med La Paz. 2016;22(2): 70-77 [Consultado el 05/08/2017]. disponible en: http://www.scielo.org.bo/ $\mathrm{pdf} / \mathrm{rmcmlp} / \mathrm{v} 22 \mathrm{n} 2 / \mathrm{v} 22 \mathrm{n} 2$ a12.pdf

16. Van Asch CJ, Luitse MJ, Rinkel GJ, Vandertweel I, Algra A, Killon CJ. Incidence, case fatality and functional outcome of intracerebral haemorrhage over time, according to age, sex and ethnic origin: a systematic review and metaanalysis. Lancet. Neurol.2010; 9(2):167-176 [Consultado el 05/08/2017]. doi: 10.1016/S1474-4422(09)70340-0.

17. Liu Y, Xu G, Wu W, Zhang R, Ying Q, Zhu W. Subtypes and one year survival of first-ever stroke in chinese patients: The Nanjing Stroke Registry. Cerebrovasc Dis. 2006,22(23):130-136 [Consultado el 05/08/2017]. disponible en: https://doi.org/10.1159/000093241

18. Castañeda-Guarderas A, Beltran-Ale L, Casma-Bustamante R, Ruiz-Grosso P, Málaga G. Registro de pacientes con accidente cerebrovascular en un hospital público del Perú. 2000-2009. Rev Peru Med Exp Salud Publica.2011.28(4):623-27 [Consultado el 05/08/2017]. disponible en: https://rpmesp.ins.gob.pe/index.php/rpmesp/article/view/425/425

19. Machado-Alva J, Machado-Duque M. Prevalencia de factores de riesgo cardiovascular en pacientes con dislipidemia afiliados al sistema de salud en Colombia. Rev Per Med Exp Salud Publica.2013;30(2):205-211 [Consultado el 15/8/2017]. disponible en: http://www.scielo.org.pe/ $\mathrm{pdf} / \mathrm{rins} / \mathrm{v} 30 \mathrm{n} 2 / \mathrm{a} 07 \mathrm{v} 30 \mathrm{n} 2 . \mathrm{pdf}$

20. Prat H. Enfermedad cerebrovascular y estatinas. Rev Chil Cardiol.2012;31(2):148-51 [Consultado el 15/8/2017]. disponible: http://dx.doi.org/10.4067/S071885602012000200010.

21. Dhiman D, Mahajan S, Sharma S, Raina R. The evolving pattern and outcome of stroke at moderate. J Neurosci Rural Pract.2018;9(1):68-72 [Consultado el 15/8/2017]. doi: 10.4103/jnrp.jnrp_207_17

22. Castilla-Guerra L, Espino-Montoro A, Fernandez-Moreno MC, Lopez-Chozas JM. Abnormal blood pressure circadian rhytm in acute ischaemic stroke; are lacunar strokes really different? Int J Stroke.2009;4(4):257-61 [Consultado el 15/8/2017]. https://doi.org/10.1111/j.17474949.2009.00314.x

23. Gibson C. Cerebral ischemic stroke: is gender important? J Cereb Blood Flow Metan.2013;33(9):1355-1381 [Consultado el 15/8/2018]. Disponible en: doi: 10.1038/ jcbfm.2013.102

24. Castillo JL, Oscanoa TJ. Dislipidemia como factor de riesgo para enfermedad cerebrovascular: estudio casos y controles. Horizonte Medico.2016;16(4): 1319 [Consultado el 15/9/2018]. disponible en: http:// www.scielo.org.pe/scielo.php?script=sci_arttext\&pi$\mathrm{d}=$ S1727-558X2016000400003

25. Chen R, Ovbiagele B, Feng W. Diabetes and Stroke: Epidemiology, pathophysiology, Pharmaceuticals and outcomes. Am J Med Sci.2016;351(4):380-386 [Consultado el 15/9/2018]. doi: 10.1016/j.amjms.2016.01.011

26. Cantu-Brito C, Ruiz-Sandoval JL, Murillo-Bonilla LM, Chiquete $\mathrm{E}$, Leon-Jimenez $\mathrm{C}$, Arauz A, et al. Manejo agudo y pronostico a un año en pacientes mexicanos con un primer infarto cerebral: resultados del estudio multicéntrico PREMIER . Rev Neurol.2010; 51(11):641-49 [Consultado el 15/9/2018]. doi: https://doi.org/10.33588/ rn.5111.2010527

27. Ariesen MJ, Claus SP, Rinkel GJ, Algra A. Risk factors for intracerebral hemorrhage in the general population: a systemic review. Stroke.2003;34(8):2060-2065 [Consultado el 15/9/2018]. disponible en: https://www.ahajournals.org/doi/10.1161/01.str.0000080678.09344.8d

28. Salvetti M, Paini A, Bertacchinini F, Aggiusti C, Stassaldi $D$, Verzeri L, et al. Therapeutic approach to hypertensi- 
ve Emergencies: hemorrhagic Stroke. High Blood Press Cardiovascular Prev.2018;25(2):191-195 [Consultado el 5/1/2019]. doi: 10.1007/s40292-018-0262-3.

29. Manning LS, Robinson TG. New Insights into blood Pressure control for intracerebral haemorrhage. Front Neurol Neuroscie.2015;37:35-50 [Consultado el 15/9/2018]. doi: $10.1159 / 000437112$

30. Sare GM, Geeganage C, Bath PM. High blood pressure in acute ischaemic stroke-broadening therapeutic horizons. Cerebrovasc Dise.2009;27 suppl 1:156-61[Consultado el 15/9/2017]. doi: 10.1159/000200454.

31. Tsivgoulis G, Katsanos AH, Butcher KS, Boviatsis E, Triantafyllou $\mathrm{N}$, et al. Intensive blood pressure reduction in acute intracerebral hemorrhage: a meta-analysis. Neurology. 2014;21:83(17):1523-9 [Consultado el 15/9/2017]. disponible en: doi: 10.1212/WNL.0000000000000917

32. Chen Z, lona A, Parish S, Chen Y, Guo Y, Bragg F, et al. Adiposity and risk of ischaemic and haemorrhagic stroke in 0.5 million Chinese men and women: a prospective cohort study. Lancet Glob Health.2018;6(6):e630-e640 [Consultado el 15/1/2019]. doi: 10.1016/S2214109X(18)30216-X

33. Bazzano L, Gu D, Whelton M, Wu X, Chen CS, Duan X, et al. Body mass index and risk of stroke among chinese men and womwn. Ann Neurol.2010;67(1):11-20 [Consultado el 15/9/2017]. doi: 10.1002/ana.21950

34. Kroll ME, Green J, Beral V, Sudlow CL, Brown A, Kirichek $D$, et al. Adiposity and ischemic and hemorrhagic stroke: prospective study in women and meta-analysis. Neurology.2016;87(14):1473-1481 [Consultado el 15/1/2019]. doi: 10.1212/WNL.0000000000003171.

35. Gloekler S, Saw J, Koskinas KC, Kleinecke C, Jung W, Nietlispach F, et al. Left atrial appendage closure for prevention of death stroke and bleeding in patients with nonvalvular atrial fibrillation. Int J Card.2017;15:234-246 [Consultado el 15/5/2019]. disponible en: doi: 10.1016/j. ijcard.2017.08.049

36. Safavi-Naeini P, Rasekh A. Closure of left atrail appendage to prevent stroke: Devices and status. Tex Heart Inst J. 2018;45(3):172-174 [Consultado el 15/2/2019]. disponible en: doi: 10.14503/THIJ-18-6693

37. Skurk C, Leistner DM, Park JW, Landmesser V. Catheter-based closure of the left atrial appendage: Stroke prevention in atrial fibrillation. Internist. 2016;57(9):844-55 [Consultado el 15/2/2018]. doi: 10.1007/s00108-016-0113-z.

38. Chanda A, Reilly JP. Lef atrial appendage occlusion for stroke prevention. Progr Cardiovasc.2017;59(6):626-635 [Consultado el 15/2/2018]. doi: 10.1016/j.pcad.2017.04.003.

39. Ruiz L, Peñaloza D. Altitude and hypertension. May Clin Proc.1977,52(7):442-5 [Consultado el 15/2/2017]. disponible en: https://pubmed.ncbi.nlm.nih.gov/875468/

40. Marmot MG, Shipley MJ, Rose G. Inequalities in death--specific explanations of a general pattern? Lancet. 1984;1:1003-6 [Consultado el 15/2/2017]. disponible en: https://www.sciencedirect.com/science/article/abs/ pii/S0140673684923377 